\title{
SOME ASPECTS OF NUMERICAL SIMULATION OF CONTROL VALVES FOR STEAM TURBINES
}

\author{
Martin HAJŠMAN, Dana KOVANDOVÁ, Richard MATAS•
}

\begin{abstract}
The contribution deals with the numerical simulation of air and steam flow through the model of control valve for steam turbines. Numerical simulations were compared with experimental measurements for the award of the same boundary conditions. Valve characteristics have been computed for individual travel heights and pressure ratios of two variants of seat inflow angle $\left(90^{\circ}\right.$ and $\left.60^{\circ}\right)$. Some other aspects are discussed in the article - comparison of the axysymmetric and $3 D$ modelling, influence of the computational domain size, comparison of characteristics for two flow media, experimental model of the valve etc. The mentioned results are important for engineering simulations and also for design of the control valves for steam turbines of the large output.
\end{abstract}

\section{INTRODUCTION}

The control valve in a steam turbine is a main component included in the turbine set. This system comprises control fittings (i.e. valves, screens or control partitions) that control the flow of steam into the turbine. The system includes valve control (i.e. servos or HP hydraulic system) which sets the valves and screens to the required flow rate and pressure of the steam. It also contains an electronic system with control software which

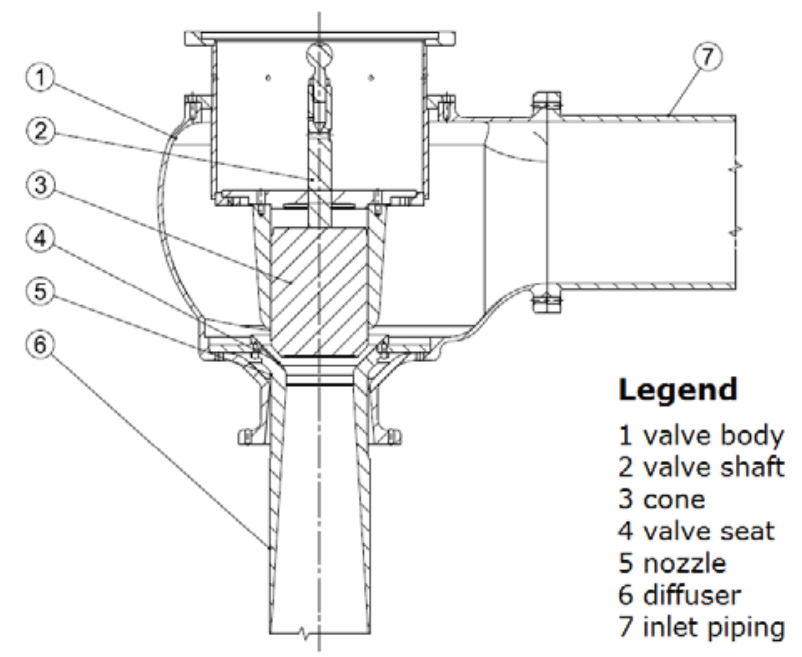
enables setting the valves, i.e. changing the steam flow into the turbine and thus changing the turbo-set output, from the control centre.

The control valve should reliably regulate the amount of steam flow and thus the turbine set output. The actual regulation is achieved by a change of the flow area between the plug and the valve seat. The control valve design is outlined in Figure 1. These valves are produced in many versions with various arrangements and plugs.

Figure 1: Chart of the control valve

\footnotetext{
- Martin Hajšman, ŠKODA POWER s.r.o., Tylova 1/57, 30128 Plzeň, Czech Republic, martin.hajsman@doosan.com

Dana Kovandová, ŠKODA POWER s.r.o., Tylova 1/57, 30128 Plzeň, Czech Republic, dana.kovandova@doosan.com

Richard Matas, Západočeská univerzita v Plzni, Univerzitní 8, 30614 Plzeň, Czech Republic, mata@ntc.zcu.cz
} 
The development of a reliable valve is not easy; it involves a combination of numerical and experimental methods. It is a topical problem of the applied research and is performed to obtain data for design of reliable control valves. More information you can find e.g. in engineering oriented article [1] or in contribution summarizing the CFD solution of problem [2].

The target of this work is accuracy verification of a CFD simulation of the flow characteristics, to explain away some questions from work [3] and evaluating an effect of the inflow angle of the valve seat (and some other parameters) on the flow characteristics to compare the results with the older [4] and new experimetal results.

\section{BALANCED-PLUG AND UNBALANCED-PLUG VALVES}

The control valves are divided into balanced and unbalanced valves according to the force necessary to change the position of the plug from the OFF position into the ON position. If the force equals force corresponding with the product of the annular area and the difference of pressures before and behind the valve, as shown in equation 1 , the valve is unbalanced. If the force controlling the plug is lower, the valve is balanced.

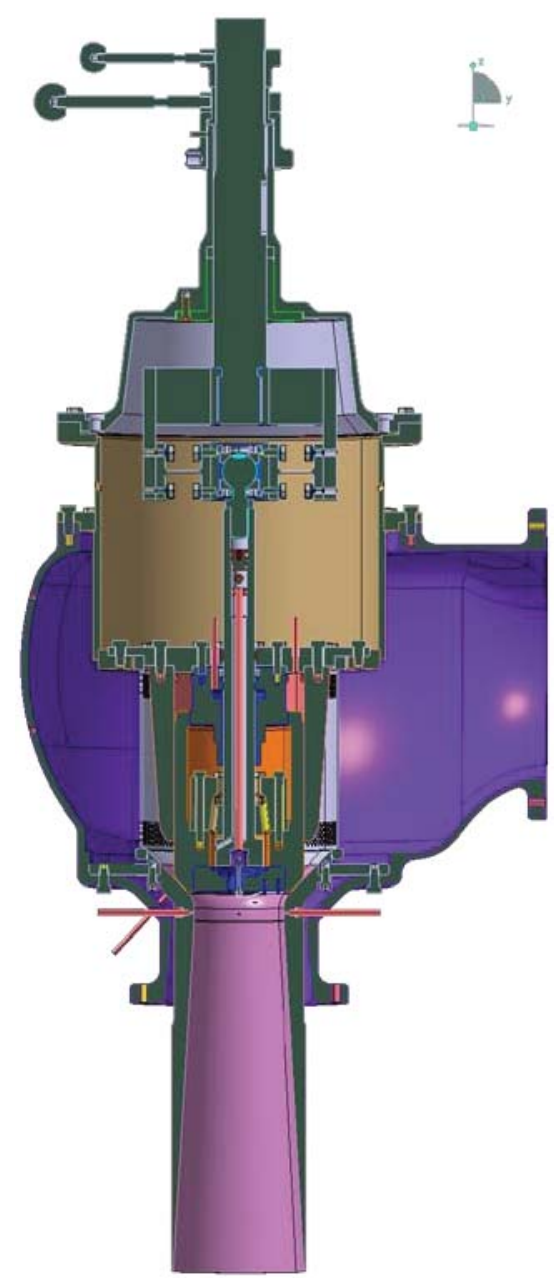

$F=\frac{\pi}{4} \cdot\left(d_{1}^{2}-d_{2}^{2}\right) \cdot\left(p_{1}-p_{2}\right)$

Valve balancing is a constructional adaptation of the valve that reduces the drive force of the valve; it involves insertion of a smaller valve into the plug of the main valve so that initially the smaller plug is moved during the opening, thus reducing the difference of pressures before and behind the valve, and only then the main plug is opened. The force necessary for lifting the small plug is low enough because the annular area is small. The force necessary for lifting the main plug is reduced because the difference of pressures is reduced.

Operations described in this work concern an experimental model valve located in the Škoda Power s.r.o. laboratory. Unlike the real steam valves, the flow medium for this valve is air. The results are transferred by means of dimensionless quantities. Valves of large performance turbines are usually designed as balanced; this balancing system and its adjustment is a subject of further research.

Also the researched valve is balanced, but the balancing was ignored in the calculations and the valve was considered unbalanced. The reason is that in the simulated higher travel of the tested valve, the flow through the balancing system has practically no effect on the overall flow and the research concentrated on comparison of the flow characteristics in higher travels. The balancing of the experimental valve can be manually closed and it can be operated as an unbalanced valve.

Figure 2: Chart of an experimental control valve 


\section{THE MAIN VALUES AND CHARACTERISTIC}

The flow through a control valve can be comprehended as a flow through a nozzle. In terms of thermodynamics, the applicable laws and principles are identical, but the geometry of the nozzle is much simpler than the geometry of the control valve. The theoretical critical mass flow, which is determined by the (2) formula, is an important value; for ideal gas, it can be replaced with the formula (3).

$$
\begin{gathered}
\dot{m}_{k r}=\left[2 \cdot\left(i_{1 C}-i_{k r}\right)\right]^{\frac{1}{2}} \cdot \rho_{k r} \cdot \frac{\pi \cdot D_{h}^{2}}{4} \\
\dot{m}_{k r}=S^{*} \cdot \sqrt{\frac{p_{0}}{v_{0}}} \cdot\left(\frac{2}{\kappa+1}\right)^{\frac{1}{\kappa-1}} \cdot \sqrt{\frac{2 \kappa}{\kappa+1}}
\end{gathered}
$$

The proportional mass flow is determined by the formula (4); it relates the instantaneous mass flow to the critical mass flow.

$$
q=\frac{\dot{m}}{\dot{m}_{k r}}
$$

The pressure ratio is a ratio between the static pressure on the outlet from the control valve and the total pressure on the valve inlet. It is determined by the formula (5).

$$
\varepsilon=\frac{p_{2}}{p_{0}}
$$

The proportional travel height is a real travel of the valve plug related to the critical diameter of the nozzle. It is determined by the equation (6).

$$
\bar{h}=\frac{H}{D}
$$

The above mentioned formulas and values are used for determination of the flow characteristics of the valve - the dependence $q$ on $\varepsilon$, i.e. a basic characteristic of the valve. The valve characteristics can be supplemented with the turbine's operational characteristics.

\section{EXPERIMENTAL AIR TUNNEL}

The model valve is located in an air tunnel of the ŠKODA POWER experimental laboratory. The air tunnel has an open cycle and the air is supplied by a radial compressor with regulation. The design and the most important parts of the air tunnel are illustrated in Figure 3.

The equipment is used for measuring flow characteristics; its design allows measurement of force characteristics, i.e. load on the plug or the shaft.

The travel height of the plug is adjusted manually by control screw positioned above the valve chamber. The control screw is coupled with a nut whose thread is screwed on the subsequent plug shaft. Turning the control screw by $360^{\circ}$ corresponds to $1 \mathrm{~mm}$ of the plug travel.

Pressures in the air tunnel in selected areas by the help of static taps are measured. Static pressures on axially symmetrical parts of the valve, such as the inlet piping, the nozzle sleeve or the valve seat are taken four times in $90^{\circ}$ on the same cross - section. 
The pressures taken from one measuring point only are subsequently merged and a hose with the resulting pressure is conducted into a pressure converter whose electric signal is processed by computer software.

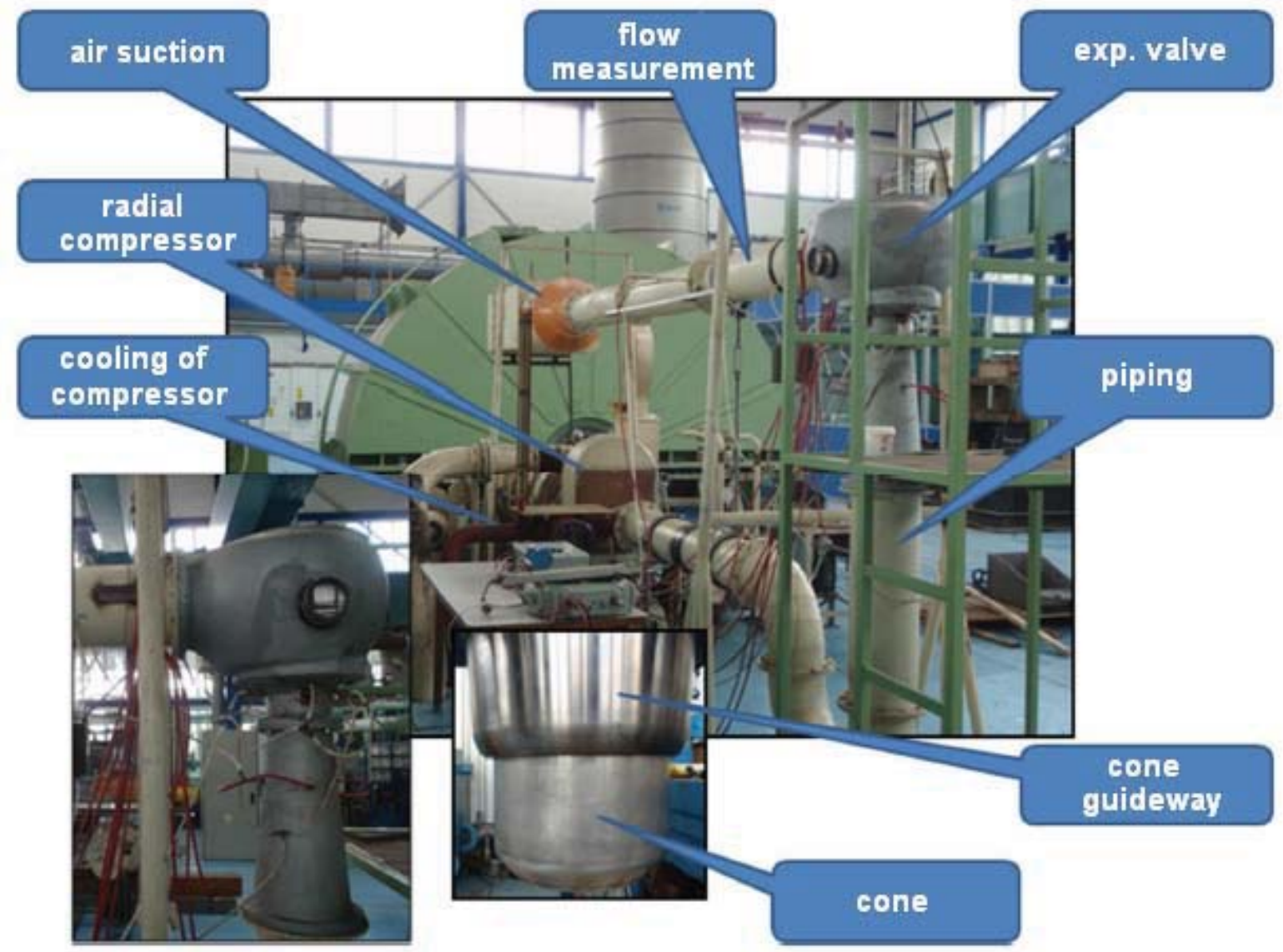

Figure 3: Air tunnel

The mass flow of the working medium is measured by a nozzle inserted in the inlet piping.

Other values related to the measurement are monitored during the experimental measurement. The current pressure in the inlet chamber of the valve and the current temperature of the inlet air are registered in each record of the measured outputs. The current critical mass flow and the pressure ratio are subsequently calculated from these values for each recorded measuring point.

The measurements were performed for a great range of operational parameters. For now, only values from $\varepsilon=0.84$ were used for comparison with characteristics obtained via CFD. Each cycle is recorded three or four times during the measurement. This method made the measurement more accurate and eliminated random errors in the measurements.

The measured data were used in a graphic chart of the flow characteristics (Figure 4). The curves corresponding to the individual travel heights illustrate the anticipated shape, i.e. a part of ellipse. The individual characteristics maintain the trend of increased proportional flow when the travel is changed to a higher level. On the other side there is a decreased trend of the proportional flow when the pressure ratio is increased. 


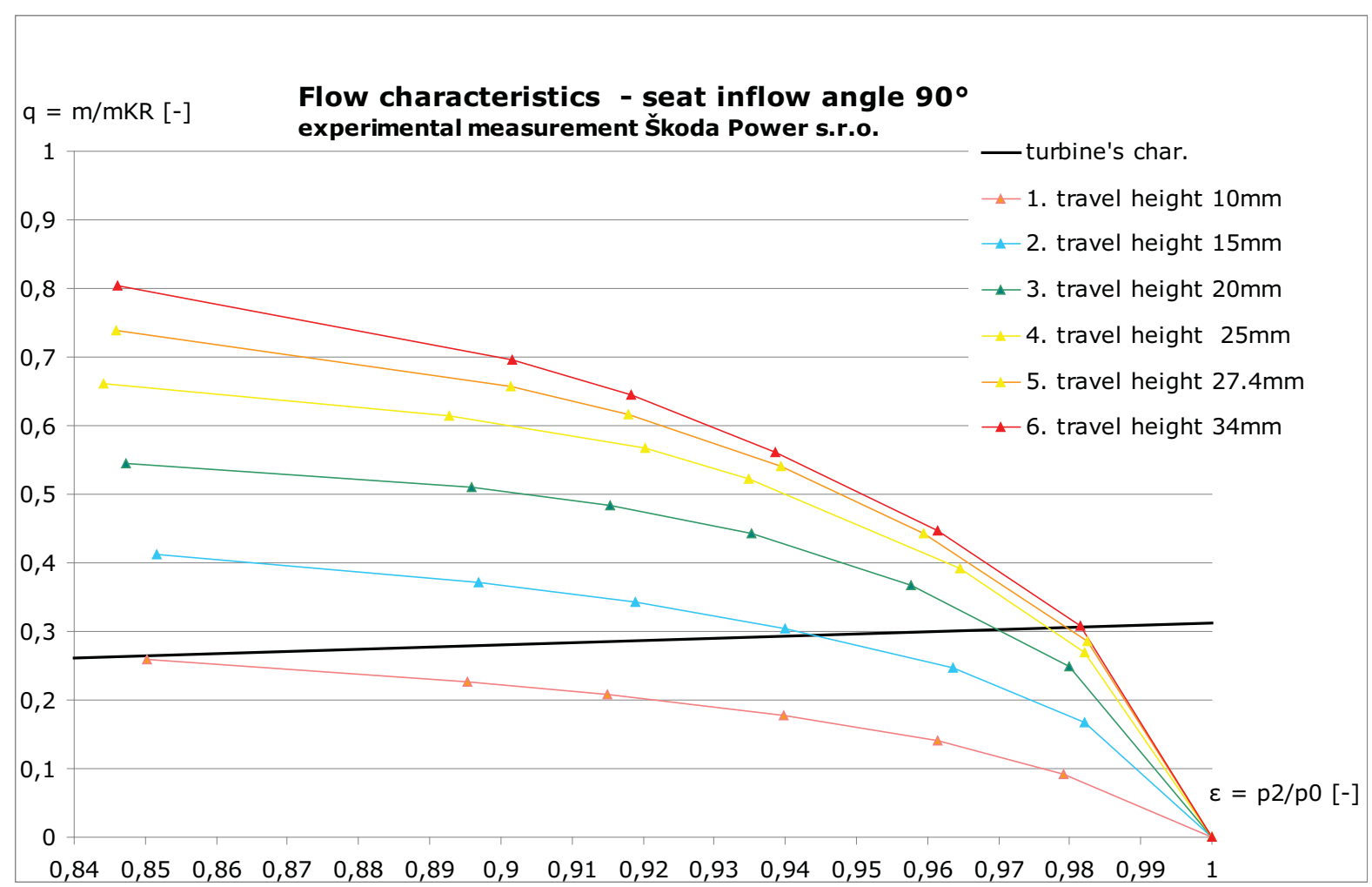

Figure 4: Chart of the flow characteristics obtained by experimental measurement

\section{NUMERICAL SIMULATIONS OF THE VALVE}

The geometry of the control valve for the simulation was adapted from 3D models of the aggregate and individual CAD elements in the Catia V5 program. The geometry was further adapted and simplified with this program. The main software tool for performing the numerical simulation of the flow was the ANSYS/FLUENT 13.0 program, the details of simulations are described in the thesis [5].

All the mathematical models were conceived without a balancing system. The desired geometry of the valve was simplified to an axially symmetrical model in the first phase. Simplification of the task into axial symmetry facilitated acquiring methodology of the calculations and testing their applicability for engineering applications, i.e. a quick check of the valve design and acquisition of flow characteristics. The 3D task represents a mathematical model based on a measured physical model. The only important difference was usage of a screen in the inlet section of the valve which was inserted into the model during the measurement.

Due to the relatively complicated geometry and flow field, the solution was complicated and frequently not very stable. The third and fourth travel heights displayed a low stability at high $\varepsilon$ values (pressure ratio), so a partially different calculation model had to be used.

To allow comparison of results, identical setting was used for all the tasks, i.e. a model of ideal gas, marginal pressure conditions and a "density based implicit" solver with adjusted second degree of accuracy. Two equation turbulence models RNG k-e and "realizable" k-e models were used. 
The computation grids were non-structured, in a axisymmetric task quad cells with a refining in the wall area for better modelling of the boundary layer, for 3D tasks tetrahedrals with prisms in the wall area. The number of cells oscillated around $20-25$ thousand in rotatively symmetrical tasks and around 2 million in 3D tasks. The geometry of the numerical model and illustration of the computational grids are given in Figure 5.

A method applicable for general as well as batch processing of the simulation was debugged for the tasks. The calculation of the given travel height started with a low $\varepsilon$ and, after stabilization of the flow pattern, the pressure ratio gradually increased. There was used a standard initialization; convergence for the selected conditions occurred after approximately 4000 iterations.
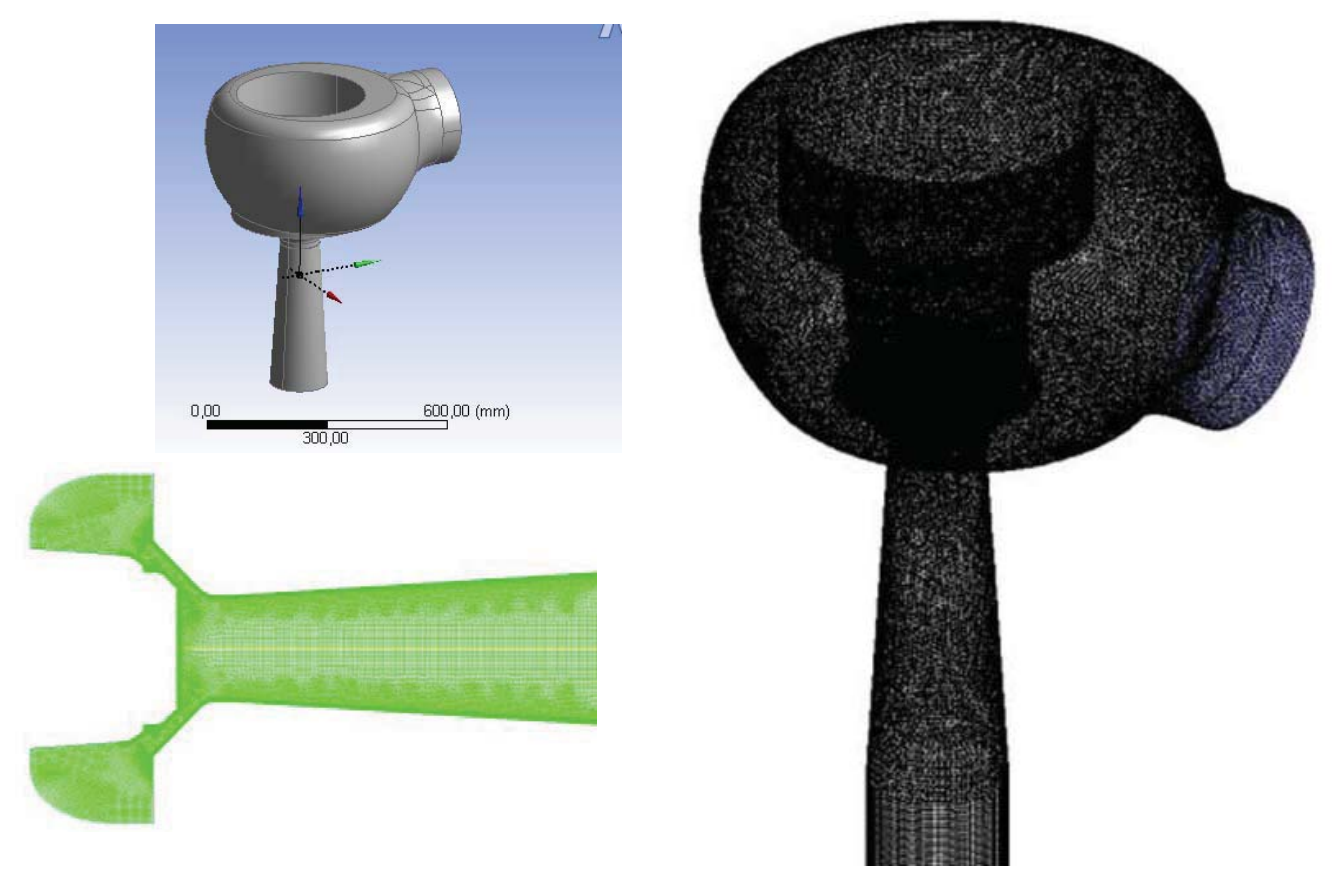

Figure 5: Geometry of the CFD model and illustration of computational grids

\section{COMPARISON OF NUMERICAL SIMULATIONS WITH EXPERIMENTAL MEASUREMENTS}

This comparison was performed in order to evaluate the accuracy of the numerical simulation results because, as it was discovered, reliable comparison of flow characteristics obtained by measurements and simulations is not available despite the existing large amount of numerical simulations and experimental measurements $[2,3]$. The numerical simulations were performed with regard to the experimental measurement and with the same marginal conditions, if possible. Although the simulation and the measurement were performed simultaneously, it was not possible to maintain absolutely identical conditions, but they were identical enough for most of the modes.

The complex comparison results of experimental measurements and numerical simulations are illustrated in Figure 6. An analysis of this chart suggests that numerical simulations can simulate the characteristics with satisfactory accuracy.

The first simulated travel height corresponds with the results most accurately as its characteristics are virtually identical. In higher pressure ratios, where the simulation is 
more demanding, the values slightly differ from the measurements. Even an experimental measurement is demanding in this sphere; it was performed for the maximum pressure ratio $\varepsilon=0.98$. However, this sphere lies beyond the presumed characteristic of the turbine.

The second travel height shows relatively low differences between the proportional mass flow of the numerical simulation curves and the curve of the experiment. In principle, the values of the individual modes in the second travel height do not differ; the curve representing the experiment shows higher mass flows than the simulation.

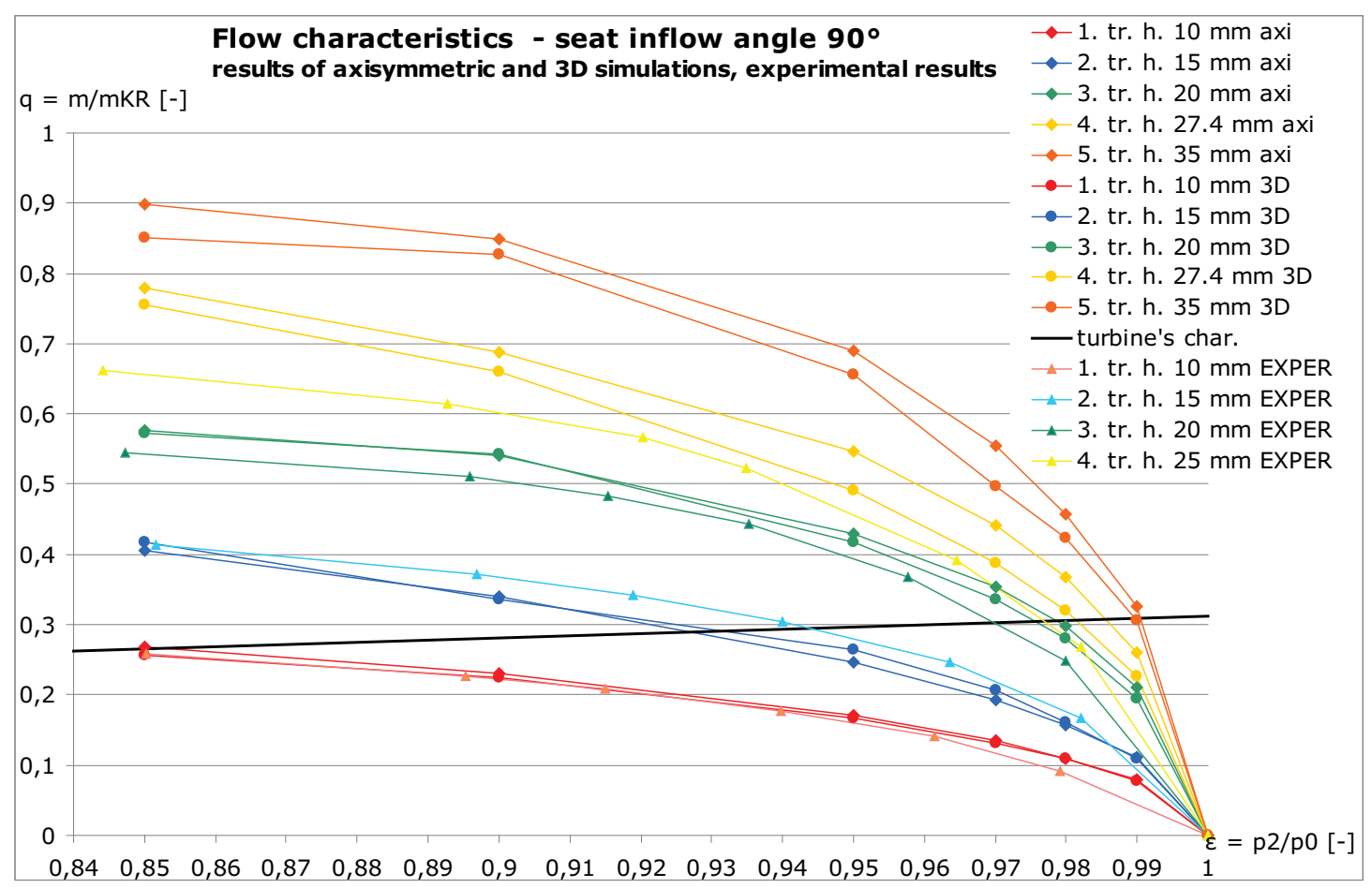

Figure 6: Flow characteristics - comparison of the simulation with the experiment

The third travel height shows approximately identical deviations as the second; the curve representing the experiment, however, shows lower values of the mass flow. Nevertheless, the proportional deviations fluctuate within $\pm 3 \div 5 \%$ in the first, second and third travel height.

At first sight, the fourth compared travel shows the largest differences of the characteristics curves. Here, however, the travel adjustment of the valve plug is not identical. The experimental measurement was performed at the travel of $25 \mathrm{~mm}$, while the simulation in the fourth travel was adjusted to the critical travel which corresponds to $27.4 \mathrm{~mm}$. With regard to this fact it is clear that the curve representing the experiment shows lower values of the mass flow than the simulation curve.

\section{NUMERICAL SIMULATIONS OF THE SEAT INFLOW ANGLE}

One of the important geometry parameters of the valve is the inflow angle of the valve seat. Currently, the geometry of the inflow angle in ŠKODA POWER s.r.o. is $90^{\circ}$. Some of the earlier works suggest that lowering this angle might produce changing of the valve losses [1]. Thus a study, in which the inflow angle was modified to $60^{\circ}$, has been 
performed; it was to compare efficiency of both versions as regards the mass flow of liquid through the valve while maintaining identical marginal circumstances. These were formed by inlet and outlet pressure and an identical clear area in the adjustment of individual travels of the plug.

The simulations of the inflow angle effects were performed as axially symmetrical and 3D models. The results of the simulations are relatively similar; they are illustrated in Figure 7 for the axisymmetric model and Figure 8 for the 3D model.

The simulations proved the effect of different geometry in both versions; it affects the resulting mass flow through the valve in all travel heights.

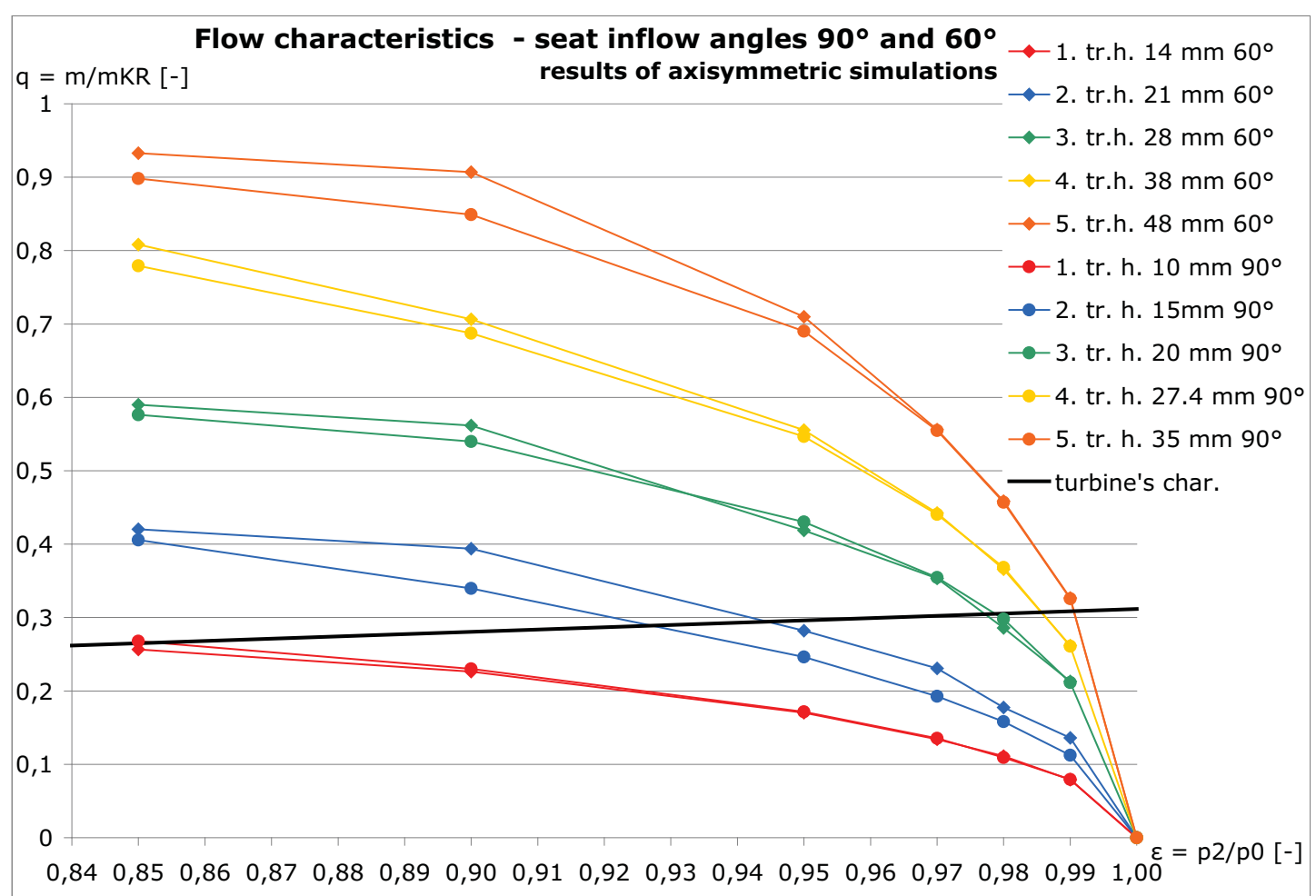

Figure 7: Comparison of characteristics of axisymmetric simulations for inflow angles $90^{\circ}$ and $60^{\circ}$

The obtained characteristics curves correspond for the axisymmetric case as well as the 3D case. Both types of calculations proved an effect of different geometry in both versions which results in a different mass flow through the valve in all the travel heights. Comparison of the mass flow in the $60^{\circ}$ and $90^{\circ}$ inflow angle proved that the mathematical model with $60^{\circ}$ produces a higher flow rate. The differences in values fluctuated within $3 \div 5 \%$ again.

In some cases, the calculation resulted in separation of the liquid flow from the wall in the sleeve area which caused a crash or instability of the calculation. This extensive separation of the flow was removed by application of a different turbulence model; the $\mathrm{k}-\varepsilon$ RNG model was replaced with the "realizable" $\mathrm{k}-\varepsilon$ model where these problems did not occur. This issue has to be tackled independently and the simulations should be confronted with experimental results in detail.

It is also necessary to include the influence of equalizing of the inlet flow through the screen, which has been omitted in the 3D models so far; this work is under preparation, just as experimental verification of the $60^{\circ}$ inflow angle. 


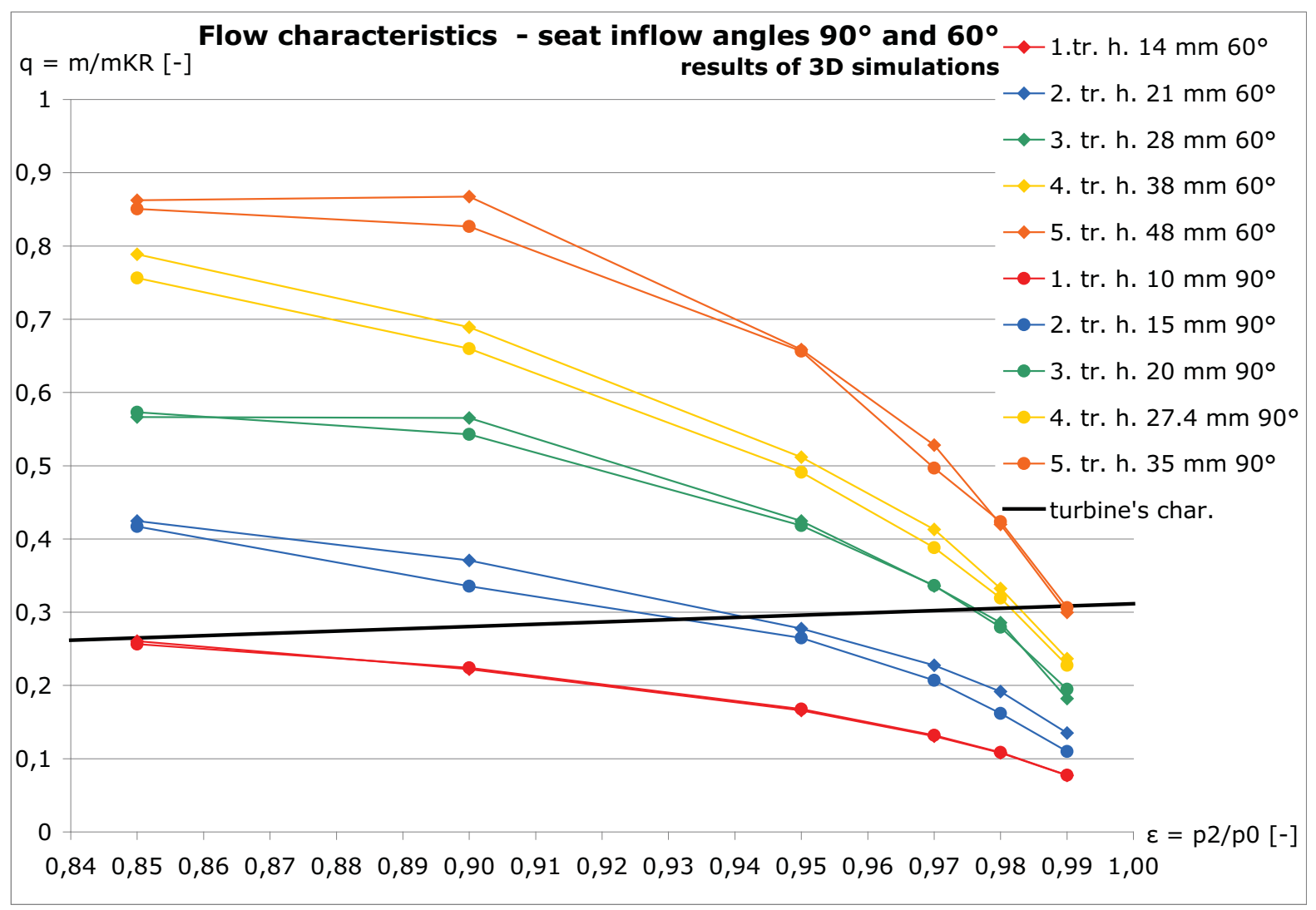

Figure 8: Comparison of characteristics of 3D simulations for inflow angles $90^{\circ}$ and $60^{\circ}$

\section{EFFECT OF SELECTED PARAMETERS ON RESULTS OF NUMERICAL SIMULATIONS}

Further results were reached in verification of certain parameters on the results of the simulations. It particularly concerns an effect of the flowing medium and the size of the numerical area on the outlet.

Ideal gas representing steam with realistic parameters for an $800 \mathrm{MW}$ turbine was used as the flowing medium for the comparison. The parameters of the medium were prepared according to the methodology for replacement of steam with ideal gas in numerical calculations where a calculation of substitute parameters of the gas from the inlet and the anticipated outlet condition was performed. Also the marginal conditions were changed to conditions of the real performance.

Although the flowing media and the marginal conditions are very different, they can be easily compared via the dimensionless quantities $-\varepsilon$ and $q$. The comparative simulations were performed for selected lifts and proportional pressures.

It is clear from the chart in Figure 9 that the flow characteristics of the steam as a flow medium in the first travel height virtually do not differ from the flow characteristic in the third travel height, but a certain deviation is evident.

This deviation, however, is not great and the simulations satisfactorily confirm justification of modelling with the selected medium and the transfer of values via dimensionless coefficients to different conditions.Although the simulations correspond with each other as regards mass flows, differences in the flow pattern are visible. In simulations with steam, the steam flow is separated from the diffuser wall in certain modes. This fact was also verified by a change of the numerical model which did not disprove the separation of the flow. In simulations of identical modes on 3D 
mathematical models, the separation of the flow did not occur, but the mass flow was virtually identical, see Figure 9.

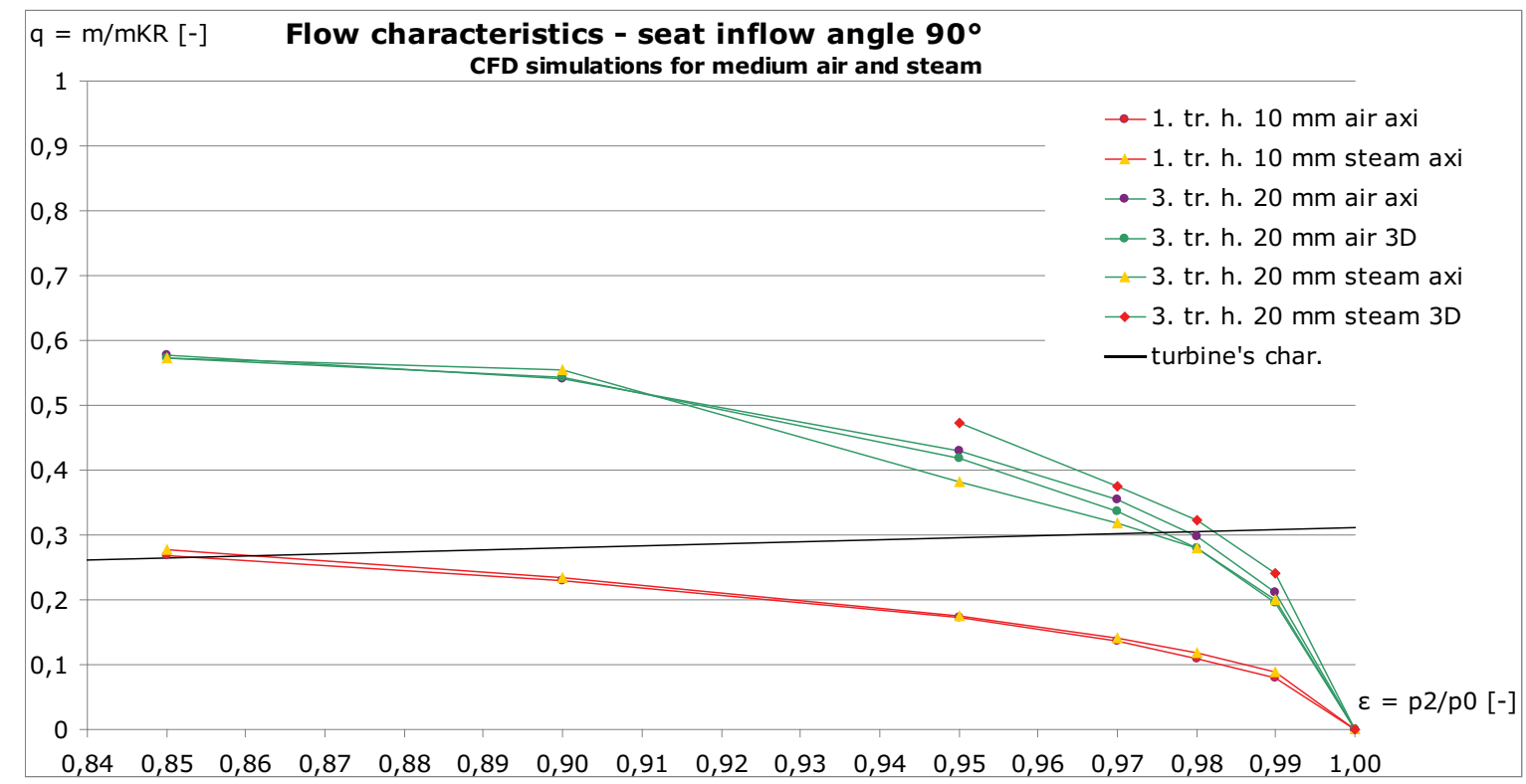

\section{Figure 9: Comparison of characteristics $\left(90^{\circ}\right)$ for two various flow media}

This fact led to adaptation of the geometrical model and prolongation of its outlet section behind the diffuser with piping. This modification was to determine the effect of the prolonged piping on the flow characteristics, or on the flow pattern.

Comparison of characteristics for air in Figure 10 shows that they are identical; the extent of the outlet area has no effect on the flow characteristics. The characteristics for alternate steam produced the same mass flows and the characteristics are mutually approximating. A small increase of the mass flow is evident in the 3D model. The characteristics are not much different from the others, the deviations fluctuated around 5 $\div 8 \%$.

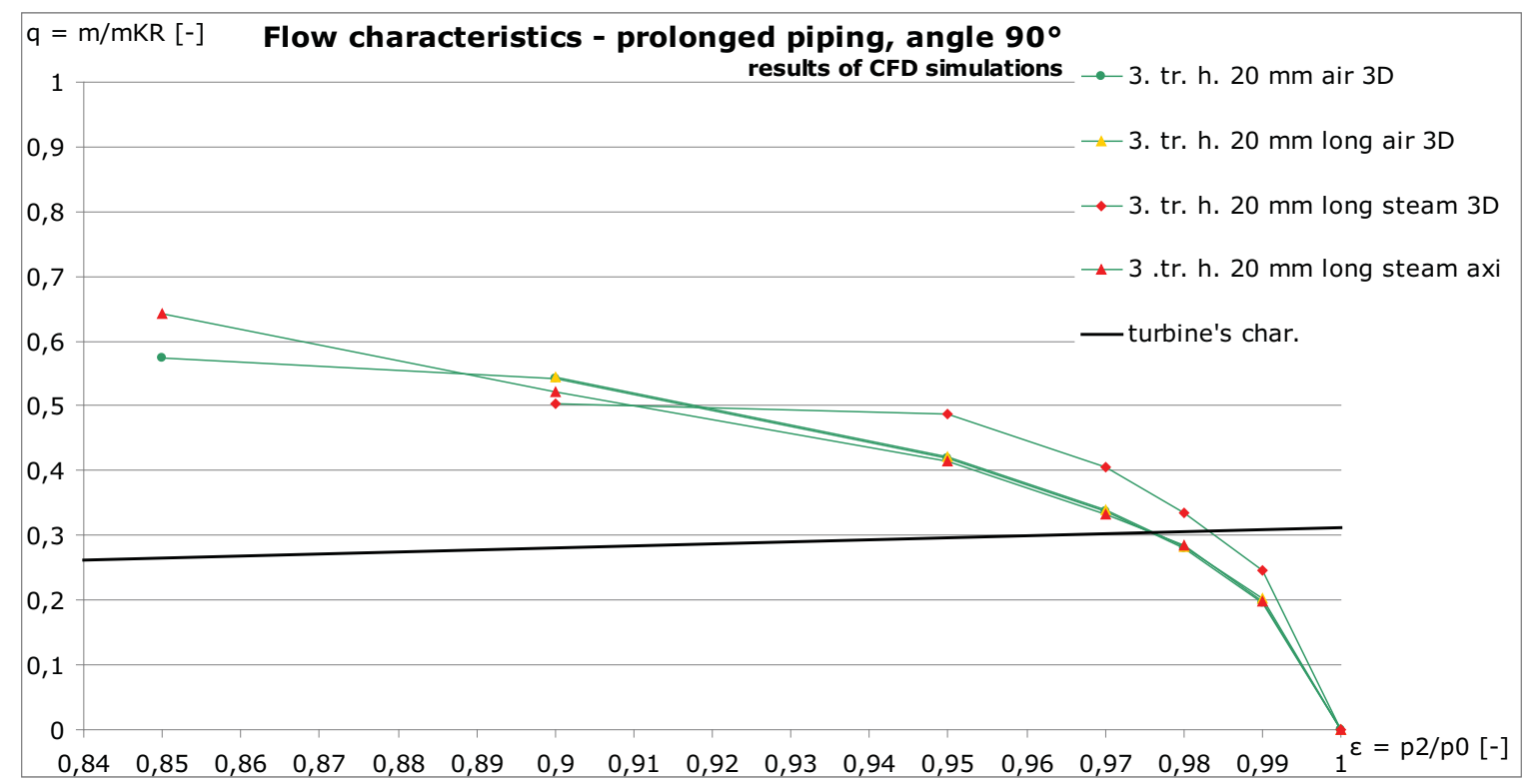

Figure 10: Characteristics of a model with prolonged piping 


\section{Conclusions}

The article summarizes results of existing works on a model of the new type of control valve for steam turbines. In the initial phase, the works concentrated on comparison of flow characteristics obtained by experiments and numerical methods. Then an effect of the seat angle on the flow characteristic and secondarily an effect of the flow medium within the calculation area of the numerical model were numerically verified. The results imply the following conclusions:

- The experimental valve of a modular design allows measurement of characteristics in a broad range of parameters; it can be operated as an unbalanced as well as balanced valve. In addition, it allows measuring pressures in the balancing system and force effects on the plug and shaft, thus significantly extending the spectrum of tasks for the actual development of the valve and for comparisons with CFD simulations.

- Comparison of flow characteristics with the experiment produced satisfactory results. The results are quite unique in their scope of comparisons of characteristics because they map a great extent of modes under known conditions. The results suggest that relatively accurate characteristics for unbalanced valves can be obtained by means of axially symmetrical models whose creations and calculations are now very simple.

- The 3D effects in the flow, e.g. its separation in the diffuser area, are more complicated. These effects are hard to predict by calculations (they depend on a correct selection of a number of parameters including a turbulence model) and their measurement is also complicated. The study also suggests that to a certain extent, they need not affect the flow characteristics, but can affect force impacts on the valve.

- The results of the simulations and produced curves proved that a change of geometry in the flow part of the valve to a newly suggested $60^{\circ}$ version can increase the flow through the valve while keeping the same flow area. The increase of the mass flow rate was approximately $3 \div 5 \%$. These results negate outcomes of an earlier report; although it contains experimentally measured flow characteristics of both versions of the inflow angle in the nozzle, it lacked a necessary detailed description of the measured conditions. Increase of the flow amount through the valve brings about reduction of thermodynamic loss of the valve, which affects the efficiency of the entire turbine set. Should the new version be put into practice, it would be necessary (besides further measurements) to adapt it to higher demands on the control equipment, i.e. the servo drive which must allow a broader extent of axial displacement.

- Testing of the effects of the flow medium confirmed an assumption regarding similarity of the valve behaviour for various flow media and the marginal conditions corresponding to conditions of a real operation of a steam turbine set with parameters of admission steam; the resulting characteristics are in line with the anticipations. Nevertheless, the simulations discovered changes in the character of the flow pattern in the diffuser.

- The output area showed practically no impact on the characteristics; partial deviations were in the flow pattern again.

- The work confirmed that numerical simulations of valves that have already been performed in large numbers are difficult to solve due to the complicated geometry and the flow patterns; no general methodology for the solution can be determined, 
so that a suitable mathematical model and methodology for calculation must be specified for each researched mode of the valve.

The work produced a number of interesting findings applicable in development of control valves. The study of this issue by means of physical as well as mathematical models will continue and concentrate on a balancing system of the valve including its numerical model. Its correct adjustment is essential for the valve control equipment because it fundamentally affects its force load.

\section{ACKNOWLEDGement}

These results were achieved in the framework of 1 M06059 project of the Výzkumná centra MŠMT programme (Research Centres, Ministry of Education, Youth, and Sports of the Czech Republic) and in specific research (project ZČU SGS-2010-040).

The access to the MetaCentrum computing facilities provided under the programme "Projects of Large Infrastructure for Research, Development, and Innovations" LM2010005 funded by the Ministry of Education, Youth, and Sports of the Czech Republic is acknowledged.

\section{REFERENCES}

[1] Yong H. Y., Kune Y. S.: Engineering analysis of mass flow rate for turbine system control and design, In Nuclear Engineering and Design 241, Elsevier, 2011, pp. 4061-4078.

[2] Matas R., Bednář L., Tajč L.: Numerical Simulations and Experiments as Modern Tools for Research of Control Valvesfor Steam Turbines. In Experimental Fluid Mechanics 2010. Liberec: Technická univerzita, 2010, pp. 398-409. ISBN: 97880-7372-670-6.

[3] Jirka L., Matas R., Tajč L.: CFD simulace proudění páry o nadkritických parametrech regulačním ventilem. In Power System Engineering, Thermodynamics \& Fluid Flow, Plzeň, Západočeská univerzita v Plzni, 2007, pp. 65-72. ISBN 978-80-7043-542-7.

[4] Zarjankin A. J.: Issledovanie merenia sdruženogo regulirujuščego klapana firmy Škoda turbiny, Research report MEI, Moscow, 1994

[5] Hajšman, M.: Výpočet průtokové charakteristiky ventilu s různými vstupními průměry sedla, diploma thesis, Západočeská univerzita v Plzni, 2011. 